\title{
Registered Nurses' Experiences with the Medication Administration Process
}

\author{
Hanna Pirinen, ${ }^{1}$ Lotta Kauhanen, ${ }^{1}$ Riitta Danielsson-Ojala, ${ }^{1}$ Johan Lilius, ${ }^{2}$ \\ Ilona Tuominen, ${ }^{2}$ Natalia Díaz Rodríguez, ${ }^{2}$ and Sanna Salanterä ${ }^{1}$ \\ ${ }^{1}$ Department of Nursing Science, University of Turku, Lemminkäisenkatu 1, 20520 Turku, Finland \\ ${ }^{2}$ Turku Centre for Computer Science (TUCS), Department of Information Technologies, Åbo Akademi University, \\ Joukahaisenkatu 3-5 A, 20520 Turku, Finland
}

Correspondence should be addressed to Lotta Kauhanen; anloka@utu.fi

Received 21 May 2015; Revised 26 August 2015; Accepted 27 August 2015

Academic Editor: Ann M. Mitchell

Copyright (C) 2015 Hanna Pirinen et al. This is an open access article distributed under the Creative Commons Attribution License, which permits unrestricted use, distribution, and reproduction in any medium, provided the original work is properly cited.

Background. Registered nurses (RNs) have a role in the medication administration process (MAP) multiple times per day in a hectic hospital environment. This requires a great deal from the RNs in order to accomplish the demanding task of avoiding adverse drug events. However, the process has not been widely studied from the nurses' perspective. Aim. The aim of this study was to describe the different stages of MAP from the RNs' perspective. Methods. A qualitative descriptive research design, with a purposive sample involving thematic interviews of $20 \mathrm{RNs}$ and questions to them in a paper form, was conducted in two medical units. Data was analyzed by using deductive content analysis. Results. The results revealed that RNs confront numerous problems such as equivocal prescriptions, problems with information technology (IT), unavailability or incompatibility of the medicines, a substantial amount of generic substitutions, and changing medicine brands. Disruptions and distraction run through each stage of the MAP, excluding prescribing. The RNs desire support in all stages of the MAP. Conclusion. There are areas to improve in each stage of the MAP from the RNs perspective. Real-time and ubiquitous documentation, along with software including the data and knowledge required in medication management, is needed.

\section{Introduction}

The medication administration process (MAP) is a complex and multistage practice in hospital settings. MAP plays a central role in nursing and is mostly managed by nurses, except prescribing that is conducted by the physicians. However, nurses are in some way involved in each stage of the MAP, including prescribing, documenting (transcribing), dispensing, administering, and monitoring. MAP is a time-consuming task, taking an estimated up to $40 \%$ of nurses' work time [1] where interruptions and distractions are common [2] which is not facilitated by the continuously increasing number of new medicines and generics [3].

In this paper, by nurse we mean registered nurses (RNs) that do not have the prescription authority. However, nurses have an essential role in prescribing. By "RNs' role in prescribing" we mean the work that nurses conduct in interpreting and understanding the prescriptions. Nurses' role in prescribing might also include suggesting medication and asking the physician to check patient's medication or to do medication changes. In addition, nurses have a responsibility to prevent, evaluate, and report drug effects, side effects, and adverse drug events. In the participating hospital nurses also program the drug administration times and document them into to the medicine software.

The complexity of the MAP exposes risks to medication errors that are surprisingly common and costly [4]. The report To Err Is Human: Building a Safer Health System estimated that, in the United States, 44,000 to 98,000 patients die annually because of medication errors, with an estimated cost of 17-29 billion dollars [5]. Many of these errors are [68] thus preventable. Moreover, developing medication safety is one of the main political objectives recognized in the European Commission [9].

Medication errors occur in all stages of the MAP in hospital settings, most frequently at administration, prescribing, 
or dispensing [10]. According to the Institute of Medicine (IOM), approximately one medication error occurs per patient per day in hospital care [4]. Nurses have an essential part in the MAP and can be considered as administrators of safety for being in the front line when medications are administered. Therefore, health-care personnel perspectives should be used when developing safer practices in hospitals [11].

\section{Background}

Medication administration is not simply a part of nurses' work but rather constitutes most of their daily work. In hospital settings, one patient may require multiple scheduled and unscheduled medication doses per day. Unscheduled doses, for example, PRN (pro re nata, as needed), occur at unplanned times and thereby create discontinuities in work. Furthermore, nurses face a complex mixture of demands related to medication administration (demands from institutional policies, technical devices, patients, the physical environment, and medications themselves) that need to be completed within the temporal structure of their shift [12]. Meanwhile, meeting these demands, nurses have an important role promoting medication safety as part of patient safety. This includes, for example, risk identification during the process, responsibility to prevent and report adverse drug events (ADEs), and learning from experience [13]. The generally regarded "five rights" principle (right patient, medication, dose, route, and time) of the MAP provides a basic standard for safe medication practice. However, focusing on the individual nurse's performance during the final stage of the process does not reflect the responsibility and accountability associated with medication administration or multidisciplinary approaches to medication management [14]. Administering medicines requires theoretical and clinical medication competence [13]. In-depth knowledge of medicines includes pharmacodynamics, therapeutic use, side effects, adverse events, and appropriateness of administering the medication considering the patient's current response to treatment [15].

The MAP is well recognized in previous studies. They have focused, for example, on nurses' requisite knowledge of the MAP, such as clinical reasoning and practices [16], pharmacological knowledge [15], and medication competence [13]. Adverse drug events $[12,15,17]$ and interruptions during the MAP [18-23] are also broadly studied. Few studies have focused on studying the MAP from the nurses' perspective, examining either the factors causing medication errors [6$8,24-26]$ or barriers for safe medication management in nursing homes [27, 28]. One recent study [29] has focused on nurses' experiences in medication safety practices. Smeulers et al. showed that nurses experience having an important role in conducting medication safety and that clinical reasoning is essential in nurses' work for safe practices [29]. However, little attention has been given to nurses' experiences in the process focusing on the problems in a broader viewpoint than safety during all stages of MAP. We also wanted to study the desired support in the MAP from RNs perspective in hospital settings. Studying the MAP from this perspective gives important information that should be considered when developing the process or applying new innovative technologies to support the process and make it run smoother and safer.

The aim of this study was to describe the MAP from the registered nurses' (RNs') perspective. Both the problems confronted and support desired in the MAP are presented, thus providing new empirical knowledge of RNs implementing the MAP on a daily basis and an insight of the possible developmental areas in the MAP. This study is part of the "Smart Medication Management" project, which aims to develop the MAP by applying new IT to support medication management. Other publications from the "Smart Medication Management" project are focusing on the "smart dosing" application that has been developed by the research group under the "Smart Medication Management" project [30, 31].

\section{Material and Methods}

A qualitative descriptive research design including thematic individual interviews and questions in a paper form was conducted.

Participants were chosen using a purposive sampling method from the population of RNs in two medical units. The inclusion criteria were participation in the MAP on the data collection day and RN willing to give a written informed consent. The eligible sample size was estimated to be 20 participants, large enough for data saturation. Saturation was reached when new data confirmed previous data without really adding new insights [32]. The final sample size of the study was 20 participants.

The settings for data collection were from two different fields of medical units in one university hospital in Finland. Both units have patients with high daily consumption of medication. Patients in these units are often elderly with chronic diseases and they come to specialized care with a need for painkillers and antiemetics in addition to the treatment medication and their regular medication. The description of the MAP in the units is shown in Figure 1. Both units used CGIs (Consultants to Government and Industry) Uranus software, where the Miranda medicine system is applied. In addition, units have nonelectronic medicine cabinets, where medicines are not single-packaged. Both units use the medicine brand names in the MAP.

3.1. Data Collection and Analysis. The data was collected in January 2013, within a two-week period, using semistructured theme interviews and questions which were filled by the participants in a paper format. Both interviews and questions were employed as a single measure in Finnish and, thus, the citations used are translations. As no validated interviews or questionnaires relevant to the topic of interest were available, the themes of the interview and the questions were developed by the research group, based on previous scientific literature. The methods of this study were founded on the following stages of the MAP: prescribing, documenting, dispensing (filling the medication trays), and administering (to patients). The researchers visited both units prior to the data collection 


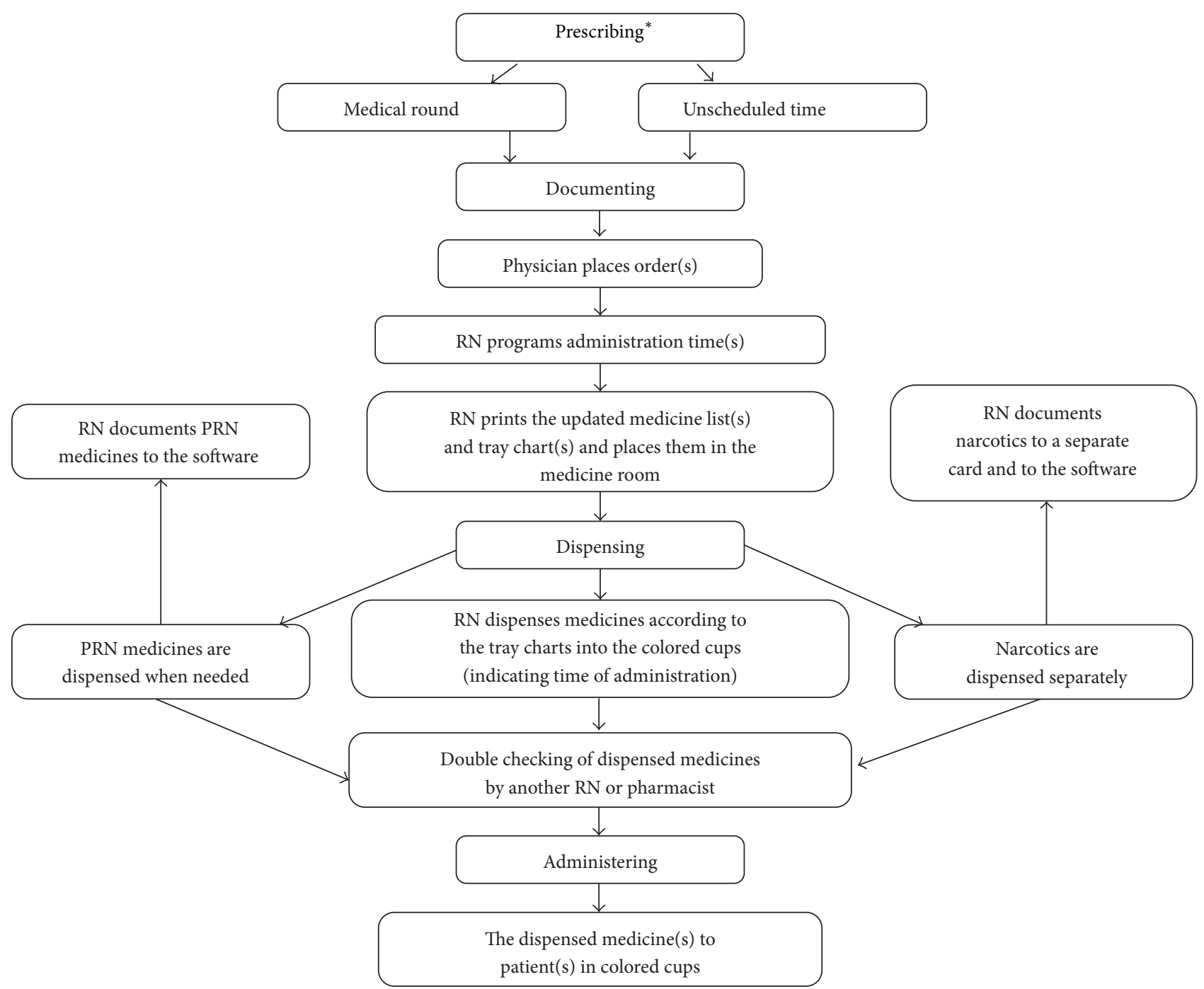

FIGURE 1: Medication administration process on the units according to the hospital policies. ${ }^{*}$ RNs role in prescribing is to interpret and to understand the prescriptions, suggest changes to medication if needed, and evaluate and report drug effects.

to present the study and inform the staff about the study. Subsequently, the staff had an opportunity to ask questions and receive answers concerning the study.

The interviews (Table 1) contained seven questions, each representing a specific theme exploring the stages of MAP. The interviews were conducted as individual in-person interviews over the participant's shift in the unit, under an environment as calm as possible, lasting 15 minutes on average. Participants were able to influence the time the interview was done. Participants' responses were typed on a laptop during the interviews.

The questions in a paper form were handed out to the participants at the beginning of their shift and they were asked to complete, after the medication administration, and return them to the researcher. The completed answers were kept safe from other participants. The questions surveyed the RNs' actions during the MAP. The questions were as follows: (1) how often do you need help in finding the wanted drug from the medicine cabinet?, (2) from what source do you check the generic substance or the changed brands of the medicines?, and (3) how often do you need to use Pharmaca Fennica when filling the medication tray?

We used both methods (questions in a paper form and inperson interviews) to enrich the data and to get more realistic descriptions of the actions during the MAP without the interviewer bias [33]. Interview is a more personal form than independently conducted questionnaire or survey, and thus, even though the questions in the paper form could have been asked during the interview, we wanted to divide the questions into a personal interview and nonpersonal paper form. One reason for this was also the practical arrangements: the questions about the actions during the MAP were supposed to be filled straight after the medication administration to ensure the best recall outcome. The interviews were conducted when the participating nurse had time. 
TABLE 1: The themes and questions of the theme interview.

\begin{tabular}{ll}
\hline Themes & Questions \\
\hline \multirow{3}{*}{ Prescribing and documenting } & $\begin{array}{l}\text { How do you experience your role as a nurse in prescribing and documentation of the medicine? } \\
\text { How does the medication software support safe medication administration? } \\
\text { What sort of problems have you confronted in the following situations: prescribing medicine and } \\
\text { documenting medicine? }\end{array}$ \\
\hline & $\begin{array}{l}\text { How do you experience dispensing (filling the medication trays)? } \\
\text { What sort of problems have you confronted when filling the medication tray and administering the }\end{array}$ \\
& $\begin{array}{l}\text { Wedicines to the patient? } \\
\text { What kind of support would you like to have when filling the medication tray and administering the } \\
\text { medicines to the patient? }\end{array}$ \\
\hline
\end{tabular}

TABLE 2: The nurses' roles in prescribing and documenting medication.

\begin{tabular}{|c|c|c|}
\hline Stage & Role & Specification \\
\hline \multirow{3}{*}{ Prescribing events } & $\begin{array}{l}\text { An advocate of } \\
\text { patients }\end{array}$ & $\begin{array}{l}\text { Informing patient of medications } \\
\text { Taking responsibility in } \\
\text { (i) prescribing situations based on their understanding of the patient's condition, } \\
\text { (ii) suggesting changes if needed in patient medications (dosing/choice of medication) }\end{array}$ \\
\hline & An active attendee & $\begin{array}{l}\text { Monitoring physician orders } \\
\text { (i) ensuring the validity of prescriptions, } \\
\text { (ii) considering patient medications as a whole (following up interactions, extra/unnecessary } \\
\text { medications), } \\
\text { (iii) reminding physician of medications that need to be discontinued, } \\
\text { (iv) informing of patients prevailing condition that needs to be considered with upcoming } \\
\text { prescriptions, } \\
\text { (v) guiding/suggesting physician to prescribe specific medicines/increase of doses }\end{array}$ \\
\hline & A passive attendee & Executing orders \\
\hline \multirow{2}{*}{$\begin{array}{l}\text { Documenting } \\
\text { prescriptions }\end{array}$} & A programmer & Programming administration times to the software \\
\hline & $\begin{array}{l}\text { A person } \\
\text { responsible/in charge }\end{array}$ & Responsible for the entire documenting process \\
\hline
\end{tabular}

The qualitative data was analyzed using deductive content analysis, where the themes and questions (the phases of MAP) guided the analysis. The analysis progressed with the following stages: preparation, organizing, and reporting. Preparation included making sense of the entire data set, selecting the unit of analysis as a unit of meaning, and analyzing the manifest content. Organizing entailed both searching relevant expressions relative to study questions and reduction of the expressions. Subsequently, grouping and abstraction of the data followed [34]. In the analysis of the questions in the paper form, quantified analysis was used to visualize the actions RNs conduct during the MAP, for example, the frequency of the use of Pharmaca Fennica. Reporting was implemented with the aid of applying Coreq's checklist [35].

3.2. Ethical Considerations. The study was licensed and approved by the authorities of the Hospital District of Southwest Finland. The study complied with a good scientific practice [36] and the ethical principles [37] within each phase. Prior to the data collection, each participant received an information leaflet and an informed consent. The data collected was handled confidentially and reported in a manner assuring anonymity.

\section{Results}

The results of the theme interviews show that RNs confront various problems, in each stage of the MAP, which interfere with their work, and have several areas they desire support in (Figure 2). The results of the perceived experiences, problems, and support desired in each stage of the MAP are described in Figure 2.

4.1. RNs' Role in Prescribing Events. RNs had different types of experienced roles in prescribing events and in documenting medications (Table 2). Mainly RNs experienced their role as substantial in prescribing events. In documenting prescriptions, RNs cited physicians as being accountable for the documenting process and RNs being accountable for programming administration times in accordance with the unit's policies. Being responsible for the whole documenting process was experienced as extremely time consuming, slow, and a task demanding accuracy, especially as orders are occasionally equivocal and ambiguous.

The problems RNs experienced in prescribing were related to prescriptions, admissions, or discharges. Prescription-related problems included equivocal and erroneous prescriptions, nonprescribed orders, crucial factors not taken 


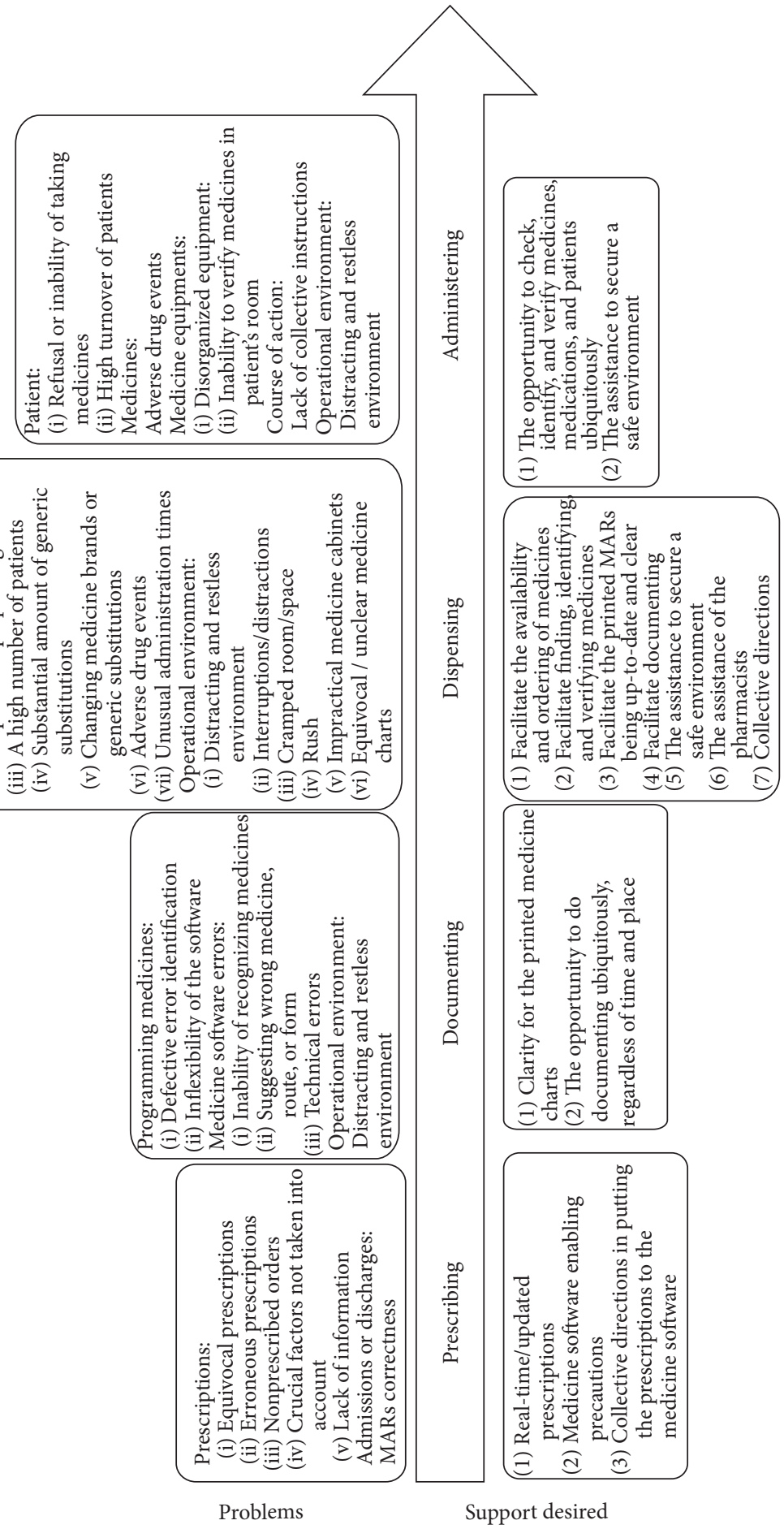

FIGURE 2: Nurses experienced problems and support desired in different stages of the medication administration process.

into account, and lack of information. Equivocal prescriptions consisted of telephone and verbal orders (e.g., mentioned when bypassing), including both incomplete prescriptions (e.g., missing strength/dose/dosing/formulation) and unclear order expiration dates (real time with last administration versus the printed medicine chart in the medicine room). RNs also experienced prescription process complicated when physicians documented medications to a wrong place in the software. That is why it was sometimes difficult for the nurses to notice new or changed prescriptions. Erroneous prescriptions comprehend errors in prescribed medicine strength or dose and prescriptions that were documented erroneously (e.g., PRN medicines prescribed to scheduled medications). Nonprescribed orders, meaning prescriptions 
that should have, but had not, been implemented by physicians (e.g., premeditations or medicines on hold that should be prescribed to continue), were experienced as problematic. Prescriptions were also experienced as difficult when crucial factors, such as interactions of prescribed medicines with patients' other medications and duplicate orders, were not taken into account, or when RNs were uninformed of new prescriptions. Verifying the correctness of medication administration records (MARs) at admission or discharge was experienced as problematic and time consuming. It was confusing for RNs to find out patients' currently active medications, latest updates, and the expiration dates of each medicine: "...every time a new patient arrives in the unit, you need to print the patient's medicine chart and find out if it's accurate...you're finding out what medicines the patient is actually taking, what dosage and when..." or "...when discharging a patient nobody goes through the patient's whole medication list. ...nurses are the ones asking what about this and that medicine...."

The support desired in prescribing was that the prescriptions were at accurate times and an updated printout was placed in the medicine rooms. Furthermore, RNs desired that the software would enable more precautions (e.g., notification of erroneous dosing) and getting collective directions for physicians when entering in prescriptions (e.g., complying with unit policies).

4.2. Documenting. In documenting, RNs experienced problems related to programming, software, and environment. Programming was problematic due to defective error identification and an unyielding structure or inflexibility of the medicine software. RNs reported that the medicine software does not report on overdoses. Programming medicines were experienced as difficult and correcting incorrect documentation was even more difficult. Additionally, errors in medicine software were experienced as problematic. RNs described the software's inability to recognize some medicines, suggesting wrong medicine or route or not finding the right form. Furthermore, RNs experienced the software as unreliable due to its technical errors: the software may throw a number of medicine strengths to the next row in the printed medicine chart, which may cause errors in dispensing and result in an error in administering. RNs also experienced printing of the charts as problematic and not systematically including all the documented medicines.

The supports RNs desired in documenting were clarity for the medicine charts and the opportunity to do documenting ubiquitously, regardless of time and place (e.g., during the medicine rounds). RNs urge that software would include all the important information needed (e.g., medicine software, patient records, medication history, prescribing and last expiration dates, and PRN medicines) in the MAP.

Medicine Software and Drug Databases in Data Transformation. Medicine software was experienced as a key component in supporting a safe MAP. RNs experienced the software supporting safety mostly with its controlling and directive documenting by reporting erroneous medication (e.g., wrong dose), verifying the right medication, and controlling the prescribing process. Furthermore, the software enhances documenting by being clear and automatic and by clarifying medicine and tray charts, leaving no possibility for misinterpretation. However, some inhibitory factors of a safe MAP came up in relation to software functionality. RNs experienced the software as clunky by describing it as slow, uncertain, inflexible, and difficult for conducting different tasks. For instance, making corrections or changes (e.g., changing the programmed timings of medicines) was difficult. RNs experienced the software lacking of technical reliability by describing it as unreliable in data transform. RNs had a great responsibility when documenting, for the software leaves room for human error. Thus, RNs have to check software functions in order to rest assured that all the prescriptions exist in the printed medicine chart and that the printout in the medicine room is up-to-date, as the software does not react if the newest chart has not been printed and placed in the medicine room. Furthermore, RNs experienced the software as unable to identify medicine errors, for not identifying or labeling interactions between different medicines or not identifying the same medicines between different brands.

With the medicine software, RNs use drug databases (Pharmaca Fennica, Duodecim) and other colleagues to support the MAP. Based on the quantified analysis of the questions in the paper form, a majority of the RNs cited employing Pharmaca Fennica daily or close to daily (12/19 and $4 / 19$, resp.) and some (3/19) weekly when dispensing. The primary source applied for finding generic medicines was Pharmaca Fennica (11/19), followed by Duodecim (7/19), and then other colleagues (3/19). The majority of RNs $(n=$ $11 / 18$ ) cited needing assistance often (close to daily or daily) for finding generic medicines from the medicine cabinet. Subsequently, assistance for this was needed occasionally (once or twice a week, $n=3 / 18$ ) and rarely (once or twice a month or less) for the RNs $(n=4 / 18)$.

4.3. Dispensing. In both units, each $\mathrm{RN}$ dispenses medicines to the patients they are in charge of over their shift. Although dispensing was experienced as laborious, difficult, time consuming, restless, and the most problematic stage of the MAP, RNs experienced it positively as a demanding task, requiring a lot of headwork, accuracy, concentration, and knowledge in order to evaluate if the medicines in the medicine and tray charts are valid. Moreover, dispensing was experienced positively as developing, educating, and maintaining skills and also as a way of gaining more patient information.

The problems experienced in dispensing were related to availability of medicines, medicines, and environment. Unavailability of medicines leads to acquisition of missing medicines, which was experienced as a burdensome and time-consuming task, involving multiple stages (e.g., requesting from another unit or pharmacy, making an order to the pharmacy). Medicine-related problems were experienced when prescribed medicines were incompatible, difficult, or impossible to dispense or to prepare the right dosage (e.g., medicine missing median line). In addition, a high 
number of patients and unfamiliarity with them, substantial amount of generic substitutions, constantly changing medicine brands, and generic substances were problematic from the RNs' perspective. Verifying (double checking) of dispensed medicines was experienced as difficult and ADEs (errors in dispensed medicines or dispensed medicines mismatching with medicine chart) were problematic for causing extra work. Additionally, unusual administration times were problematic to remember alongside routinelike times. Furthermore, RNs experienced several problems related to operational environment. RNs experienced that the medicine room was often cramped and not peaceful enough to concentrate on the demanding task, when people are coming and going; or the $\mathrm{RN}$ is interrupted for having to conduct another task (e.g., patient alarms). In addition, impractical properties of the medicine cabinets impeded finding the medicines, by being impractical and disorganized. The printed medicine charts were experienced as equivocal for the unclear markings of medicines on hold and especially when tray charts were folded in half on the medication tray. Furthermore, RNs experienced it as problematic when other colleagues forgot to print and take the updated medicine chart to the medicine room. Additionally, PRN medicines, only visible in the software, were experienced as laborious and difficult for having to leave the medicine room to check those if needed.

RNs desired the most support in the dispensing stage. First, RNs desired assistance with finding, identifying, and verifying medicines or generic substances easier and faster as the following citation shows:

\section{"If it would be possible to have a device where you could quickly, at a glance, see the appearance of the medicine and the medicine box."}

Support was also desired for verifying dispensed medicines and to have certainty that the prescribed medicines are dispensed. RNs cited quicker facilitation if an electrical device would notify them of medicines that need to be dispensed. The bar code scanning method [38] was also mentioned as one solution in facilitating the verification of the right patient or medications. Second, RNs desired up-to-date and clearly printed medicine charts. Printing was problematic, for both not being certain if the chart in medicine room is valid in accordance with the latest prescriptions or changes and the prescriptions being unclear for the different handwritten markings on them. Third, support with documenting was desired, especially with narcotic medicines dispensed separately and documented twice over. RNs expressed a desire to document dispensed medicines to one place only and that the documented data would be updated in real time with patients' MARs. Fourth, RNs expressed needing assistance in securing a safe and peaceful dispensing space to eliminate distractions. Other elements RNs desired for support were facilitating the availability and ordering process of medicines and getting help from the pharmacist in general and when problems arise with dispensing the medicines. Finally, RNs desired collective directions for dispensing (e.g., colored medicine cups indicating administering times and dispensing on collective times).
4.4. Administering. The problems $\mathrm{RNs}$ experienced in administering were related to patients, medicines, medicine equipment, course of action, and environment. Patient-related problems come up when a patient refuses or is unable to take medication. For instance, a patient may refuse to take generic substances and desire medicines with specific brands; or they may have swallowing problems. Additionally, a high turnover of patients was experienced as problematic and possibly a leading cause for the medicine-related problems experienced, such as administration to a wrong patient. The medicine devices were dysfunctional from the RNs' perspective. For instance, medicine cups tip over easily, especially when having a high amount of medicines in them. In addition, the inability to recheck or verify medicines in patients' rooms was also problematic. RNs thought it would be convenient to be able to check medicines regardless of time or place, as the following:

"...if a patient wants to go through all the
medicines in the medicine cup, and there are
medicines that I don't identify, it would be nice to
get help for that...that I could check the medicines
right there (in the patient's room) by what the
medicine looks like...I would be able to go through
the medicine one by one with the patient..."

Lack of collective instruction was experienced as problematic for causing differences in practices. Unspecified or incomplete instructions caused uncertainty, for instance, on whether medicines are administered to patients in isolation. Some RNs leave the medicine(s) on the "block," while others are uncertain when or if the medicines were administered at all. Furthermore, RNs experienced administering medicines in a calm environment without any distractions to be impossible.

The desired support in administering was uniform: RNs desired to be able to check and verify medicines ubiquitously (e.g., in patients' rooms). RNs desired to identify and verify medicines and check their purpose of use if a patient desires to look through them and also to be able to check patients' MARs, regardless of time or place:

\section{"... a device where you could check a patient's PRN medicines at a glance."}

Assistance in securing a peaceful environment was also desired in this stage.

\section{Discussion}

5.1. Summary of Evidence. The aim of this study was to describe the MAP from the RNs' perspective, by describing the RNs experienced problems and desired support in the MAP. This study shows that RNs have various roles in the MAP and RNs confront numerous obstacles impeding their work, making the MAP problematic. Yet, medication administration was experienced mostly as a positive task.

Problems in the MAP were experienced throughout the process. Problems related to RNs' role in prescribing events, such as interpreting equivocal prescriptions, may expose risks, 
as recognized in previous studies where nurses identified the illegible handwriting of physicians $[6,8,25]$, complex orders (unclear expression and illegible writing) [7], and poor physician communication [26] as being conditions causing medication errors. Furthermore, RNs experienced the disconnection between medicine software and printed medicine charts as problematic. Moreover, this could cause errors if $\mathrm{RNs}$ forgot to print the updated chart for the medicine room, when many new orders are given at unscheduled times without informing the RNs, or when physicians put orders into the wrong data field, thus making it even harder for RNs to notice these changes as found in this study. Tiredness and exhaustion $[6,8,25]$ could easily affect forgetting to print the updated medicine chart, thus causing errors. RNs desired the prescriptions to be up-to-date and in real time, meaning that when the physician makes an order in the software, it would be updated in the medicine room as well, without having to print a new chart. As found in this study, erroneous prescriptions were also found problematic in the study of Mayo and Duncan [6], where RNs experienced drug errors occurring when a physician prescribes a wrong dose. Moreover, Gill et al. [39] found that nurses identified prescribing errors as the second most contributing factor to medication errors. Thus, as the results show, RNs desired medicine software that enables precautions (e.g., notifications of erroneous dosing). Another desire was to have collective directions, for instance, considering the times when physicians made orders. However, this might be impossible due to constantly changing conditions of patients. Other problems RNs experienced in their role in prescribing (e.g., medicine chart correctness) are concurrent with how RNs experienced their role of an advocate, an active attendee, and a person in charge. In this respect, RNs are patients' gatekeepers and front line warriors when it comes to the patients' safety and best interests. Although physicians are in charge of prescribing and documenting prescriptions, the RNs' involvement is substantial in both actions. Occasionally, RNs have to exceed their duties for physicians not complying with unit policies when documenting prescriptions. As the results show, nurses' roles in medication management cannot be overemphasized [14].

The problems RNs experienced in documenting were mostly related to the dysfunction of the software. The results suggest that the current IT does not adequately support RNs' work in the MAP. The software is prone for human error and its usability is inflexible. RNs desired new IT to be applied for the MAP that would enable ubiquitous documenting and clarity for the printed medicine charts, where one solution could be the use of electronic charts only. Dispensing was found as the most problematic stage in the MAP. Unavailability of medicines was experienced as problematic in dispensing. Similarly, Vogelsmeier et al. [27] recognized the absence of needed medications as a barrier to safe medication. Furthermore, a high volume of generic substitutions was experienced as problematic and was concurrent with the support desired: facilitating finding, identifying, and verifying medicines. This is significant when taking into consideration the study of Jones and Treiber [25], who found that large number of medicines scheduled, at peak times, causes medication errors frequently. It can be assumed that a large number of generic substitutions may cause even more errors. Moreover, constantly changing medicine brands and generic substitutions, which were experienced as problematic in the study, can be seen in other studies as well. Both Mayo and Duncan [6] and Ulanimo et al. [8] discovered that drug errors occur when there is confusion between two drugs with similar names. In the study of Tang et al. [7], nurses identified unfamiliarity with medications (e.g., new drug name, similar drug names or packages, seldom-used drug, and unclear labeling) as a condition that results in medication error. Additionally, Jones and Treiber [25] found that nurses perceived look-alike or sound-alike drugs as a cause of frequent medication errors. According to health professionals, deviations may occur as a result of confusing medications due to the similar names and packaging of medicines; therefore, avoiding confusing medicines is a significant factor in preventing ADEs [11]. Also, the desired aid of pharmacists can play a key role in preventing the medication errors reaching patients [24]. The problems acknowledged in dispensing demonstrate the need for the consideration of applying new medication-dispensing technology to support RNs in dispensing, which nurses have also perceived as a factor in reducing medication errors [25].

Finally, problems and support desired in administering medicines were discovered. Patient-related problems in this study are similar to Dilles et al. [28], who found the patients' right to refuse care as a barrier to safe medication management. Furthermore, the inability to verify medicines while administering was experienced as problematic. Hence, the chance for ubiquitous usage of medicine software was a uniform desire on behalf of RNs. A lack of collective instructions in administering, discovered in this study, is concurrent with [28], who discovered insufficient guidelines being a barrier to safe medication management.

Operational environment-related problems were experienced as problematic in each stage of the MAP, excluding prescribing. Previous studies $[18,19]$ show that interruptions during medication administration are common and can lead to human error. In the study of Mayo and Duncan [6], RNs ranked the top perceived causes of drug errors where distraction of others (coworkers, patients, or events) was ranked as the second-highest cause of medication errors. Similarly, other studies $[8,25,39]$ have recognized interruptions and distractions as contributing factors of medication errors from the nurses' perspective. Additionally, in the study of Tang et al. [7], nurses identified having to solve other problems while administering drugs as the leading condition that resulted in medication error. On the strength of the study and previous studies, it is highly important, and a crucial factor, to enable concentration on the task in preventing deviations in the MAP, as Härkänen et al. [11] have also found in their study. Clarity for the problematic, unclear medicine charts was also desired, which is found to be a barrier to safe medication from nurses' perspectives [28]. Hence, forgetting to print an updated chart should be replaced with electronic charts, thus erasing possible human error. 
5.2. Study Strengths and Limitations. The two methods used for data collection and two persons analyzing the data (data and researcher triangulation) strengthen the validity of the results. Additionally, motivation and enthusiasm of the participants can be considered as strength. However, this study has some limitations. The data was collected using interviews and questions in a paper form developed for this study on the basis of previous research but not validated in earlier studies. This research is limited to one hospital and two units only; thus, the results are not generalizable. However, they are mainly in line with results in several previous studies. The study provided new insights precisely from the RNs perspective of MAP and their actions during MAP and put together previous research, hence creating a comprehensive whole of the MAP.

The study is valuable in that it describes RNs' personal experiences with the MAP. This study identified both a number of problems confronted and the support desired in the process, hence aiding the development of a more safe and flowing process. Furthermore, this study provides a framework for nurse managers to utilize the results of this study when planning the safety of the MAP in a hospital care setting.

\section{Conclusion}

Recognizing RNs' concerns and recognizing desired support in the MAP are important first steps in improving the MAP. The problems recognized in this study are in line with both the support RNs desire and previous studies considering the causes of medicine errors. The results of this study show that there are improvements required in the MAP for RNs to be able to conduct safe medication administration and maintain patient safety. RNs have plenty of ideas of how the MAP could be developed, and their views should be taken into account when developing the process. Further research is needed when developing and applying new technology solutions to assist medication management in order to be the most convenient from users' perspectives.

\section{Conflict of Interests}

The authors declare that there is no conflict of interests regarding the publication of this paper.

\section{Authors' Contribution}

Hanna Pirinen, Lotta Kauhanen, Riitta Danielsson-Ojala, Johan Lilius, Ilona Tuominen, Natalia Díaz Rodríguez, and Sanna Salanterä designed the paper. Hanna Pirinen, Lotta Kauhanen, and Riitta Danielsson-Ojala collected the data. Hanna Pirinen and Lotta Kauhanen analyzed the data. Hanna Pirinen and Lotta Kauhanen helped in writing the original paper. Riitta Danielsson-Ojala, Johan Lilius, Ilona Tuominen, Natalia Díaz Rodríguez, and Sanna Salanterä helped in paper review and comments. All authors have read and approved the final paper.

\section{Acknowledgments}

The study was supported by Special State Grant EVO (no. 13190, Turku University Hospital) funding. The authors thank all the RNs from the two units who participated in this study.

\section{References}

[1] G. Armitage and H. Knapman, "Adverse events in drug administration: a literature review," Journal of Nursing Management, vol. 11, no. 2, pp. 130-140, 2003.

[2] C. Conrad, W. Fields, T. McNamara, M. Cone, and P. Atkins, "Medication room madness: calming the chaos," Journal of Nursing Care Quality, vol. 25, no. 2, pp. 137-144, 2010.

[3] European Medicines Agency, "EMA Recommends 81 Medicines for Marketing Authorisation in 2013," http://www.ema.europa .eu/ema/index.jsp?curl=pages/news_and_events/news/2014/01/ news_detail_002006.jsp\&mid=WC0b01ac058004d5c.

[4] P. Aspden, J. Wolcott, J. L. Bootman, and L. R. Cronenwett, Preventing Medication Errors: Quality Chasm Series, Institute of Medicine of the National Academies, The National Academies Press, Washington, DC, USA, 2007, http://www.nap.edu/ openbook.php?record_id=11623\&page $=111$.

[5] L. T. Kohn, J. M. Corrigan, and M. Donaldson, To Err Is Human: Building a Safer Health Care System, National Academy Press, Washington, DC, USA, 2000.

[6] A. M. Mayo and D. Duncan, "Nurse perceptions of medication errors what we need to know for patient safety," Journal of Nursing Care Quality, vol. 19, no. 3, pp. 209-217, 2004.

[7] F.-I. Tang, S.-J. Sheu, S. Yu, I.-L. Wei, and C.-H. Chen, "Nurses relate the contributing factors involved in medication errors," Journal of Clinical Nursing, vol. 16, no. 3, pp. 447-457, 2007.

[8] V. M. Ulanimo, C. O'Leary-Kelley, and P. M. Connolly, "Nurses' perceptions of causes of medication errors and barriers to reporting," Journal of Nursing Care Quality, vol. 22, no. 1, pp. 28-33, 2007.

[9] European Commission \& DG Health and Consumer Protection, Patient Safety-Making It Happen! Luxembourg Declaration on Patient Safety, European Commission \& DG Health and Consumer Protection, 2005, http://ec.europa .eu/health/ph_overview/Documents/ev_20050405_rd01_en.pdf.

[10] D. H. Cousins, D. Gerrett, and B. A. Warner, "A review of medication incidents reported to the National Reporting and Learning System in England and Wales over 6 years (20052010)," British Journal of Clinical Pharmacology, vol. 74, no. 4, pp. 597-604, 2012.

[11] M. Härkänen, S. Saano, H. Turunen, and K. VehviläinenJulkunen, “Terveydenhuollon henkilöstön näkemykset lääkityspoikkeamien estämisestä erikoissairaanhoidossa," Hoitotiede, vol. 25, no. 1, pp. 49-61, 2013.

[12] B. M. Jennings, M. Sandelowski, and B. Mark, “The nurse's medication day," Qualitative Health Research, vol. 21, no. 10, pp. 1441-1451, 2011.

[13] V. Sulosaari, R. Suhonen, and H. Leino-Kilpi, "An integrative review of the literature on registered nurses' medication competence," Journal of Clinical Nursing, vol. 20, no. 3-4, pp. 464-478, 2011.

[14] J. Choo, A. Hutchinson, and T. Bucknall, "Nurses' role in medication safety," Journal of Nursing Management, vol. 18, no. 7, pp. 853-861, 2010. 
[15] K. H. Frith, E. F. Anderson, F. Tseng, and E. A. Fong, "Nurse staffing is an important strategy to prevent medication errors in community hospitals," Nursing Economics, vol. 30, no. 5, pp. 288-294, 2012.

[16] G. L. Dickson and L. Flynn, "Nurses' clinical reasoning: processes and practices of medication safety," Qualitative Health Research, vol. 22, no. 1, pp. 3-16, 2012.

[17] L. A. Treiber and J. H. Jones, "Devastatingly human: an analysis of registered nurses' medication error accounts," Qualitative Health Research, vol. 20, no. 10, pp. 1327-1342, 2010.

[18] A. D. Biron, M. Lavoie-Tremblay, and C. G. Loiselle, "Characteristics of work interruptions during medication administration," Journal of Nursing Scholarship, vol. 41, no. 4, pp. 330-336, 2009.

[19] E. S. Elganzouri, C. A. Standish, and I. Androwich, "Medication administration time study (mats): nursing staff performance of medication administration," Journal of Nursing Administration, vol. 39, no. 5, pp. 204-210, 2009.

[20] J. I. Westbrook, A. Woods, M. I. Rob, W. T. M. Dunsmuir, and R. O. Day, "Association of interruptions with an increased risk and severity of medication administration errors," Archives of Internal Medicine, vol. 170, no. 8, pp. 683-690, 2010.

[21] L. M. Kosits and K. Jones, "Interruptions experienced by registered nurses working in the emergency department," Journal of Emergency Nursing, vol. 37, no. 1, pp. 3-8, 2011.

[22] S. Buchini and R. Quattrin, "Avoidable interruptions during drug administration in an intensive rehabilitation ward: improvement project," Journal of Nursing Management, vol. 20, no. 3, pp. 326-334, 2012.

[23] M. Tomietto, A. Sartor, E. Mazzocoli, and A. Palese, "Paradoxical effects of a hospital-based, multi-intervention programme aimed at reducing medication round interruptions," Journal of Nursing Management, vol. 20, no. 3, pp. 335-343, 2012.

[24] I. S. Sanghera, B. D. Franklin, and S. Dhillon, "The attitudes and beliefs of healthcare professionals on the causes and reporting of medication errors in a UK Intensive care unit," Anaesthesia, vol. 62, no. 1, pp. 53-61, 2007.

[25] J. H. Jones and L. Treiber, "When the 5 rights go wrong. Medication errors from the nursing perspective," Journal of Nursing Care Quality, vol. 25, no. 3, pp. 240-247, 2010.

[26] J. Maiden, J. M. Georges, and C. D. Connelly, "Moral distress, compassion fatigue, and perceptions about medication errors in certified critical care nurses," Dimensions of Critical Care Nursing, vol. 30, no. 6, pp. 339-345, 2011.

[27] A. Vogelsmeier, J. Scott-Cawiezell, and D. Zellmer, "Barriers to safe medication administration in the nursing homeexploring staff perceptions and concerns about the medication use process," Journal of Gerontological Nursing, vol. 33, no. 4, pp. 5-12, 2007.

[28] T. Dilles, M. M. Elseviers, B. Van Rompaey, L. M. Van Bortel, and R. R. V. Stichele, "Barriers for nurses to safe medication management in nursing homes," Journal of Nursing Scholarship, vol. 43, no. 2, pp. 171-180, 2011.

[29] M. Smeulers, A. T. Onderwater, M. C. B. van Zwieten, and H. Vermeulen, "Nurses' experiences and perspectives on medication safety practices: an explorative qualitative study," Journal of Nursing Management, vol. 22, no. 3, pp. 276-285, 2014.

[30] N. D. Rodríguez, J. Lilius, R. Danielsson-Ojala et al., "Can IT health-care applications improve the medication tray-filling process at hospital wards? An exploratory study using eyetracking and stress response," in Proceedings of the 16th IEEE International Conference on e-Health Networking, Applications and Services (Healthcom '14), pp. 423-428, October 2014.
[31] N. D. Rodríguez, N. A. Khan, R. Danielsson-Ojala et al., "Smart dosing: a mobile application for tracking the medication trayfilling and dispensation processes in hospital wards," in 6th International Workshop on Intelligent Environments Supporting Healthcare and Well-Being (WISHWell '14), J. C. Augusto and K.-H. Wolf, Eds., Lecture Notes in Computer Science, pp. 1-10, Springer, 2014.

[32] U. H. Graneheim and B. Lundman, "Qualitative content analysis in nursing research: concepts, procedures and measures to achieve trustworthiness," Nurse Education Today, vol. 24, no. 2, pp. 105-112, 2004.

[33] D. C. Hildum and R. W. Brown, "Verbal reinforcement and interviewer bias," The Journal of Abnormal and Social Psychology, vol. 53, no. 1, pp. 108-111, 1956.

[34] S. Elo and H. Kyngäs, "The qualitative content analysis process," Journal of Advanced Nursing, vol. 62, no. 1, pp. 107-115, 2008.

[35] A. Tong, P. Sainsbury, and J. Craig, "Consolidated criteria for reporting qualitative research (COREQ): a 32-item checklist for interviews and focus groups," International Journal for Quality in Health Care, vol. 19, no. 6, pp. 349-357, 2007.

[36] Finnish Advisory Board of Research Integrity, "Responsible conduct of research and procedures for handling allegations of misconduct in Finland," in Guidelines of the Finnish Advisory Board on Research Integrity 2012, 2013, http://www.tenk.fi/sites/tenk.fi/files/HTK_ohje_2012.pdf.

[37] Declaration of Helsinki, World Medical Association Declaration of Helsinki, 2009, http://www.laakariliitto.fi/liitto/ etiikka/helsingin-julistus/.

[38] S. B. Fowler, P. Sohler, and D. F. Zarillo, "Bar-code technology for medication administration: medication errors and nurse satisfaction," Medsurg Nursing, vol. 18, no. 2, pp. 103-109, 2009.

[39] F. Gill, V. Corkish, J. Robertson, J. Samson, B. Simmons, and D. Stewart, "An exploration of pediatric nurses' compliance with a medication checking and administration protocol," Journal for Specialists in Pediatric Nursing, vol. 17, no. 2, pp. 136-146, 2012. 


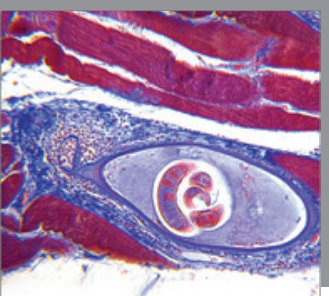

Gastroenterology

Research and Practice
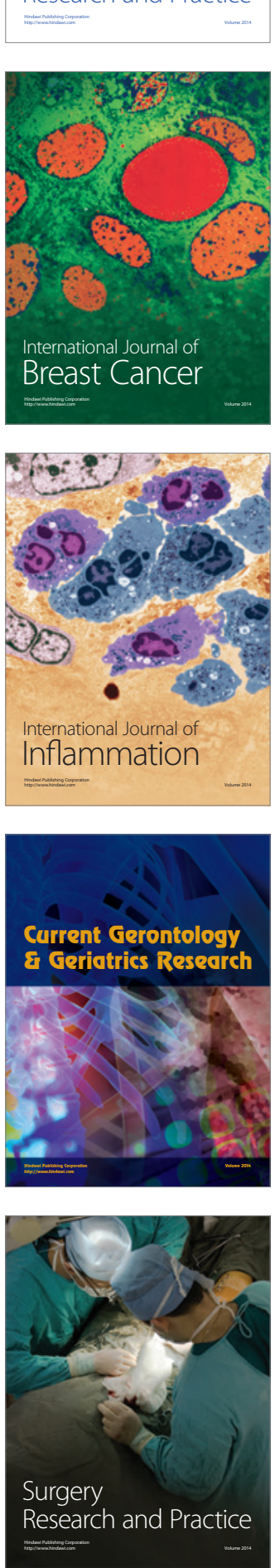

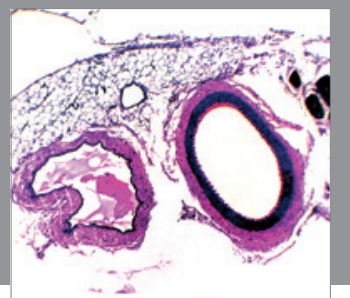

International Journal of Hypertension
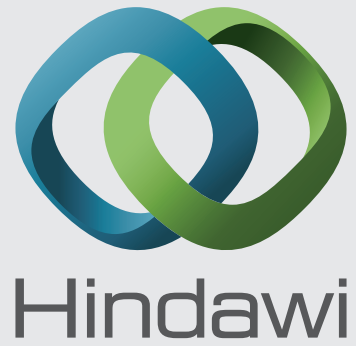

Submit your manuscripts at http://www.hindawi.com
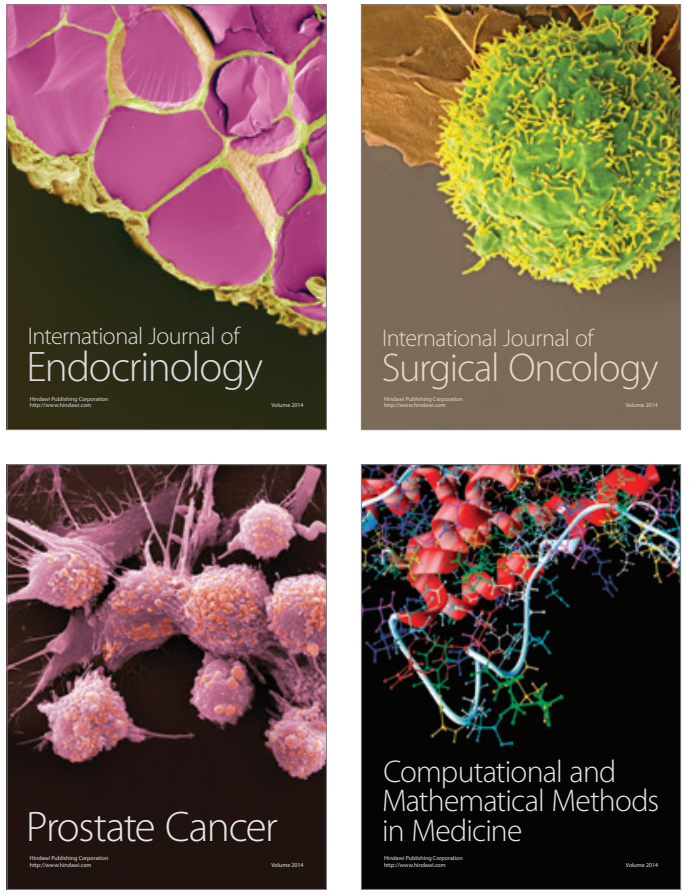
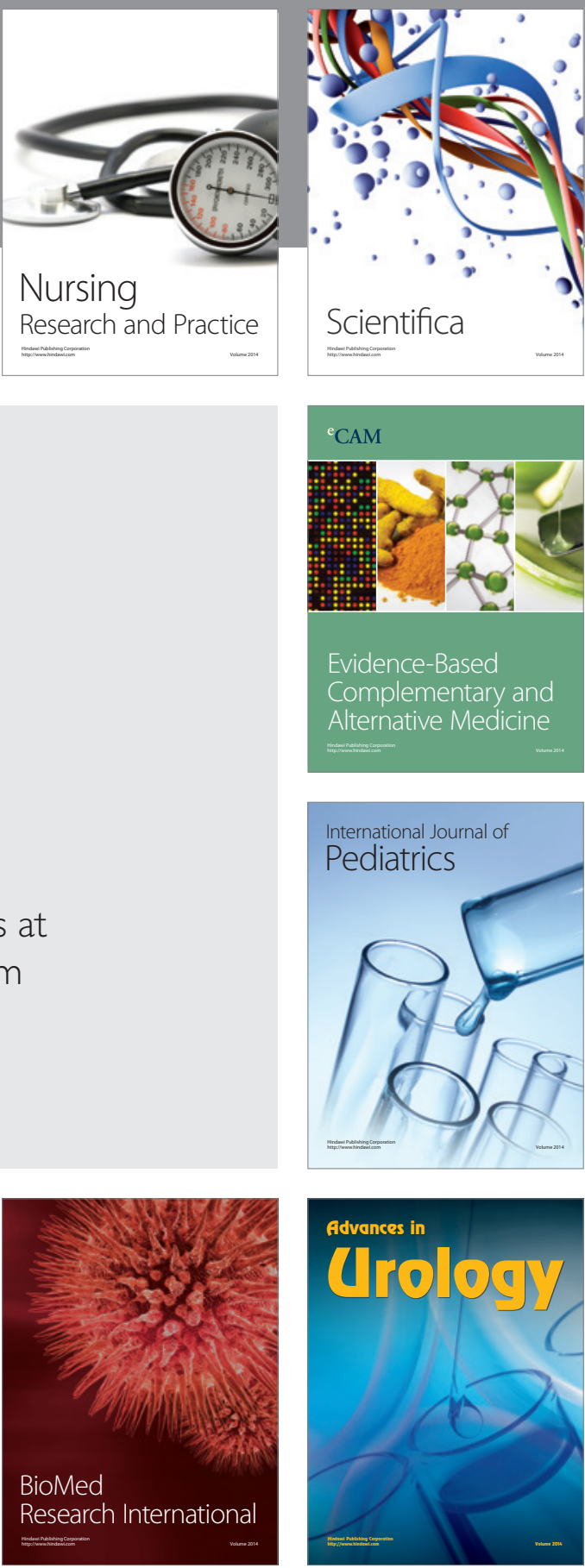

Nursing

Research and Practice

Scientifica

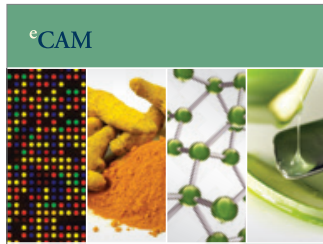

Evidence-Based

Complementary and Alternative Medicine
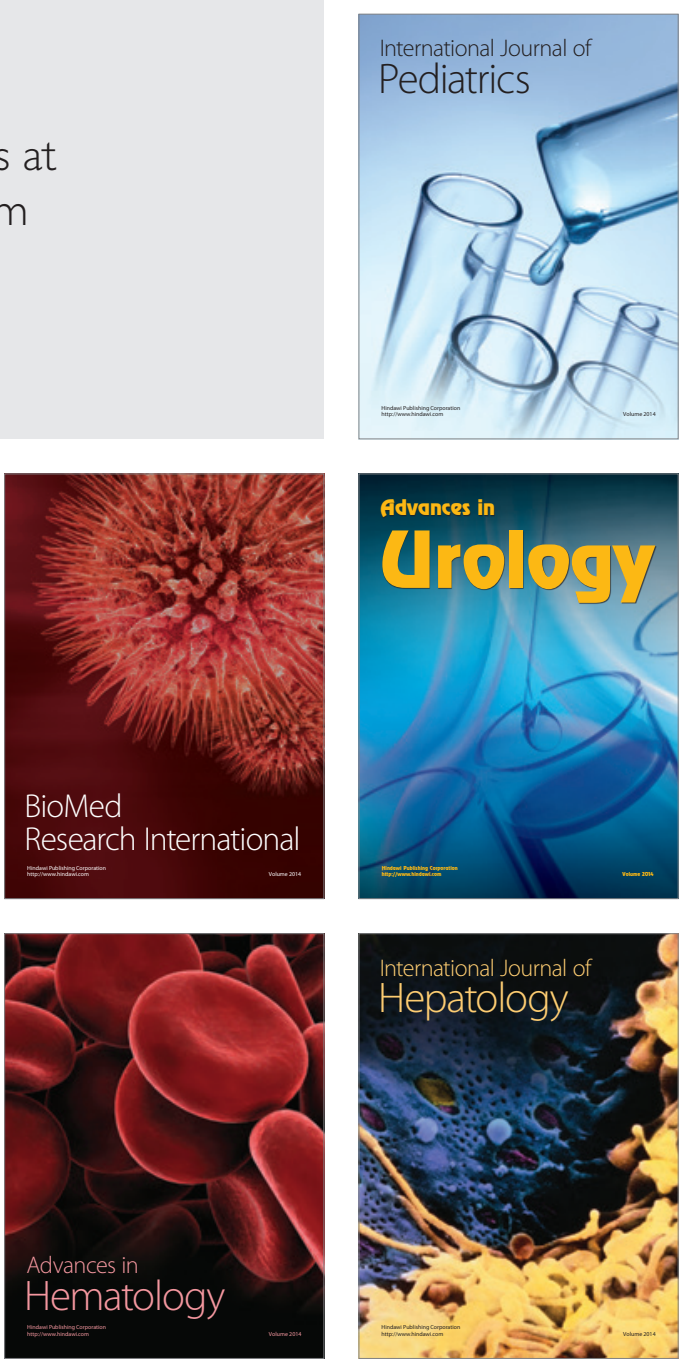